\title{
Editorial
}

by Eva Maria Pfostel

The Review of Studies on Sustainability has always given great value to the multisectoral and multidisciplinary approach.

This issue was divided into two sections.

The first section is dedicated to essays that from different points of view, deal with the issues of Sustainability: territorial governance, third sector bodies, energy efficiency, geothermal energy, finance and new models of food consumption.

The second section, instead, is entirely dedicated to a themed issue where the legal approach to Sustainability is central. The care of this section has been entrusted to Prof. Pylypenko, whose editorial describes all the presented essays.

\section{First section}

The territories can contribute to the pursuit of sustainable development, overcoming the segmented approach to well-being, that is, "governing the three fundamental economic functions (allocation of capital stocks, accumulation of the same, distribution of the wealth produced) through a" nonasymmetrical "conjugation of principles of ethics, economic efficiency, intra-generational and inter-generational equity". This is addressed by the authors Gian Paolo Cesaretti, Irene Paola Borrelli and Immacolata Viola, in the paper "Sustainability, Territories and Circular Economy".

With the issuance of the Third Sector Code, the Italian legislator seems to have laid the foundations for the construction of a third sector right, understood as a sub-system with respect to the group of rules that regulate both profit and non-profit private organizations. In the paper "Reform of the Third Sector in the perspective of Sustainable Development", the author, prof. Marina Romano, emphasizes the innovations that highlight cultural change in relation to the driving role of the third sector in the perspective of sustainable development.

In the paper "Long-term financial sustainability: an evaluation methodology with threats considerations”, the authors, Guryanova Lidiya, Bolotova

Rivista di Studi sulla Sostenibilità, (ISSN 2239-1959, ISSNe 2239-7221), 2020, 1

DOI: 10.3280/RISS2020-001001 
Olena, Gvozdytskyi Vitalii, Sergienko Olena, propose a new evaluation methodology for managing corporate systems. This makes it possible to identify the class of not only current, but also forecast situations for a given horizon of proactive management and to choose an adequate preventive strategy.

Gianfranco Franz's essay "For a New Ecological Thought” reconstructs the trajectory of the notion of sustainability, starting from the founding moments of ecological thinking and the environmental crisis. The author highlights the substantial failure of culture and policies for sustainable development and the limited contribution of the humanities, social and arts disciplines to the construction of a different relationship between human beings and the environment.

The reduction of fossil energy consumption and useful interventions to improve the energy efficiency of the European agro-food system, are the themes at the center of the work, "Energy consumption and improvement of energy efficiency for the European agricultural-food system” proposed by Greco Carlo, Campiotti Alessandro, De Rossi Patrizia, Febo Pierluigi, Giagnacovo Germinia. A list of "key performance indicators" for the fruit and vegetable sector is reported, useful for comparative analyzes aimed at identifying methods of managing the energy and the most responsive technologies to improve the energy efficiency of the agri-food sector.

The document "Incentive-Based Policy to Promote the Production of Geothermal Power from Carbon Tax Scheme: A Case of Indonesian CGE Model" examines the impact of the taxation of carbon taxation on fossil fuels and the tax is then assigned to encourage the supply of geothermal electricity. The results show that carbon taxation can effectively reduce national greenhouse gas emissions.

Local sustainable development projects can be promoted through participatory processes involving local citizens and organizations as stakeholders, following multi-stakeholder approaches. The article "The bottom-up approach is teetering. When sustainability does not match public participation: the case of an urban re-greening project in small town in a Northern Italy”, presented by Andrea Pronti, analyzes the case of an innovative participatory sustainability project in a country in northern Italy.

Waste and food losses are the challenges faced in the work "Food losses and food waste: The Industry 4.0 opportunity for the sustainability challenge”, by the authors Roberto Ruggieri, Giuliana Vinci, Marco Ruggeri, Henry Sardaryan, who investigate the opportunities that innovations present in Industry 4.0 can offer opportunities to reduce food waste and loss, as well as to adopt a healthier and more sustainable lifestyle. 
Agriculture is one of the most impactful sectors for environmental pollution and food choices have a direct influence on our ecological footprint. In the essay "Hemp (Cannabis Sativa L.): Sustainability and challenges for the food sector", a review of the literature is proposed, with a specific focus on the challenges regarding the sustainability of cultivation, innovations and the market for hemp and its products.

The authors, Alessandra Jacumizzi and Laura Emma Milani, study how the impacts of climate and demographic changes require a rethinking of food production and consumption, in the paper "Insects at the table: what consumers know". It seems that with a view to sustainable development, the inclusion of insects in the diet is a good solution. Therefore, the way opens up for an in-depth analysis of the information that must be provided to the consumer so that he can make an informed choice and towards a design of information and communication campaigns suitable for this purpose.

\section{Second section}

I would like to thank Prof. Pylypenko for the care of the editorial of the second section and all the authors who offered their valuable contribution in it.

My sincere thanks to all the authors of the first and second section for contributing to the realization of this issue. 